\title{
Fetal Electrocardiography and Deep Learning for Prenatal Detection of Congenital Heart Disease
}

\author{
Rik Vullings ${ }^{1}$ \\ ${ }^{1}$ Eindhoven University of Technology, Eindhoven, The Netherlands
}

\begin{abstract}
Congenital heart disease (CHD) is one of the main problems that can occur during pregnancy. Annually, 300.000 babies die during pregnancy or infancy because of CHD. Early detection of CHD leads to reduced mortality and morbidity, but is hampered by the relatively low detection rates (i.e. $<60 \%$ ) of current CHD screening technology. This detection rate could be improved by complementing echocardiographic screening with assessment of the fetal electrocardiogram (ECG).

In this study, the fetal ECG was measured noninvasively, with electrodes on the maternal abdomen, in almost 400 fetuses, $30 \%$ of which had known CHD. The fetal ECG measurements were processed to yield a 3dimensional fetal vectorcardiogram. A deep neural network was trained to classify this fetal vectorcardiogram as either originating from a healthy fetus or CHD. The network was evaluated on a test set of about 100 patients, showing a CHD detection accuracy of $76 \%$. Non-invasive fetal electrocardiography therefore shows clear potential in diagnosis of CHD and should be considered as supplementary technology next to echocardiography.
\end{abstract}

\section{Introduction}

Congenital heart disease (CHD) is the most common severe congenital anomaly worldwide with a reported incidence of eight per 1000 births [1]. Every year, around 1.35 million newborns are born with CHD. A third of these defects is critical and approximately $4.5 \%$ of fetuses with CHD die in-utero and $21 \%$ die postnatally [2]. Those who survive with CHD have a nine-fold increased risk of intellectual disability [3].

The timely detection of CHD has important advantages. It allows parents to prepare for the arrival of a sick child. Furthermore, it facilitates appropriate changes in obstetric and neonatal management, including intra-uterine therapy, planning of the delivery in a centre with the required neonatal and cardiothoracic surgical care facilities, and timely treatment after birth. It has been demonstrated that prenatal diagnosis of CHD increases survival rates and decreases long-term morbidity [4]-[9] .

The antenatal detection of CHD is currently done via ultrasonic examination. In most developed countries, a standard anomaly screening is performed at around 20 weeks of gestation. The assessment of the fetal heart is an important component of this screening. However, despite mass screening, about half of severe CHD are still missed [10]-[14].

Improvement of prenatal CHD detection rates is thus urgently needed and might come from the use of noninvasive fetal electrocardiography. Electrocardiography is used worldwide as a relatively simple tool to diagnose heart disease in adults and children. Recent advances in fetal electrocardiography have made it possible to acquire a high-quality fetal electrocardiogram (ECG) at gestational ages of about 18 weeks and higher [15], [16]. Moreover, it has been shown that the waveform of the fetal ECG changes in CHD [17]-[21].

This study aims to detect such CHD-induced changes in the fetal ECG waveform and use fetal electrocardiography for the detection of CHD at around 20 weeks of gestation. However, to be able to detect ECG abnormalities, it must first be known what a normal fetal ECG at 20 weeks gestation looks like. Unfortunately, due to the different cardiovascular circulation, the fetal ECG is expected to be different from that of newborns. At the moment, virtually no guidelines exist for the interpretation of fetal ECG signals. To further complicate things, non-invasive fetal ECG measurements are performed with electrodes positioned on the maternal abdomen. Since the fetus can take any orientation in the uterus, and hence with respect to these electrodes, the ECG morphology can vary within and between recordings due to differences in the fetal orientation.

To circumvent the lack of clinical guidelines for assessing CHD in non-invasive fetal ECG recordings, this study aims to use deep neural networks to classify ECGs as either originating from a healthy fetus or from a fetus with CHD, irrespective of the fetal orientation. 


\section{Data}

Non-invasive fetal ECG measurements were performed at six different medical centres in the Netherlands. Healthy women of at least 18 years of age who had a singleton pregnancy were enrolled in the study, after having given written informed consent. The study population was divided into two groups, a cohort of healthy fetuses and a group of fetuses with CHD. The presence or absence of CHD was determined during conventional ultrasonic examination and confirmed after birth. In total 386 measurements were collected, 266 from the healthy group and 120 from the CHD group. Baseline characteristics were the same for both groups.

The CHD group consisted of various types of CHD, including septal defects (ventricular and atrioventricular), conotruncal defects (e.g. transposition of the great arteries or tetralogy of Fallot) or hypoplastic single ventricle defects. Nevertheless, in this study a binary classification was used: either CHD or not.

\section{Methods}

\subsection{Fetal electrocardiography}

Measurements were performed with a prototype fetal monitoring system (Nemo Healthcare, the Netherlands). Six bipolar electrophysiological signals were recorded and stored for offline use. The duration of each measurement was approximately 30 minutes.

The recorded signals were processed on a PC to suppress interferences from maternal ECG [22], mains powerline [23], and electromyographic noise. Subsequently, fetal QRS complexes were identified [24] and used as anchor points for segmenting the signals into individual ECG complexes.

For each complex, a 3-dimensional vectorcardiogram (VCG) was calculated [25] and corrected for possible fetal movement [26]. For the movement-corrected VCGs, an ensemble average was calculated over 250 heartbeats to further suppress interferences and yield a relatively highquality fetal VCG. A schematic representation of these processing steps is provided in Fig. 1.

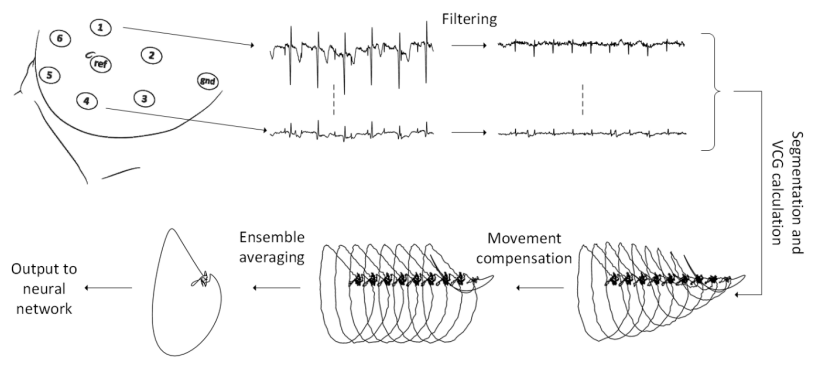

Figure 1: Schematic representation of the signal processing steps that were used to obtain a relatively high-quality fetal vectorcardiogram.

The fetal VCG with its associated label (i.e. 0 for healthy, 1 for CHD) were used as input to a deep neural network that was subsequently trained to classify fetal VCGs as either originating from a healthy heart or CHD.

Prior to training the network, data from 99 patients (50 healthy, $49 \mathrm{CHD}$ ) were excluded to test the trained network.

\subsection{Deep neural network}

The proposed network consists of 6 convolutional layers with residual connections and leaky rectified linear units as activation functions. After each layer, a maxpooling operation was used to downsample the VCG. After the sixth layer, two fully connected layers were used, followed by a softmax function. The Adam optimizer was used for training the network, with dropout as regularization method.

Data augmentation was used in the training set for two reasons. Firstly, a training set of almost 300 recordings is typically too small to yield a generalizable result. Secondly, and probably most importantly, due to the small size of the training set, there could be a bias between fetal orientation and presence of CHD. To illustrate this: if the same fetus would be recorded in cephalic and in breech position, one would expect the fetal VCG to be rotated by 180 degrees. In case there would be relatively more fetuses with CHD in breech position than in cephalic position, the network might learn an incorrect, non-existing association between the orientation of the VCG and the presence of CHD.

Hence, random rotations of the VCG were performed to augment the training set by tenfold. Another fourfold augmentation was obtained by adding random, Gaussiandistributed noise to the fetal VCGs.

During training, $10 \%$ of the training data was use as validation set to enable early stopping.

\subsection{Performance metrics}

After convergence of the network parameters during training, the final network was applied to the test set of 99 patients. No data augmentation was used for this test set. Classification accuracy was calculated as:

$$
\text { Accuracy }=\frac{T P+T N}{T P+F P+T N+F N},
$$

where $T P$ is the number of true positives, $T N$ the number of true negatives, $F P$ the false positives, and $F N$ the false negatives.

\section{4. $\quad$ Results}

Figure 2 and 3 show examples of the orthogonal 
projections of the fetal VCG that is obtained by the fetal electrocardiography signal processing. Both examples are taken from the training set. The VCG in Figure 2 is from a healthy fetus, while the fetus depicted in Figure 3 was diagnosed with tetralogy of Fallot.

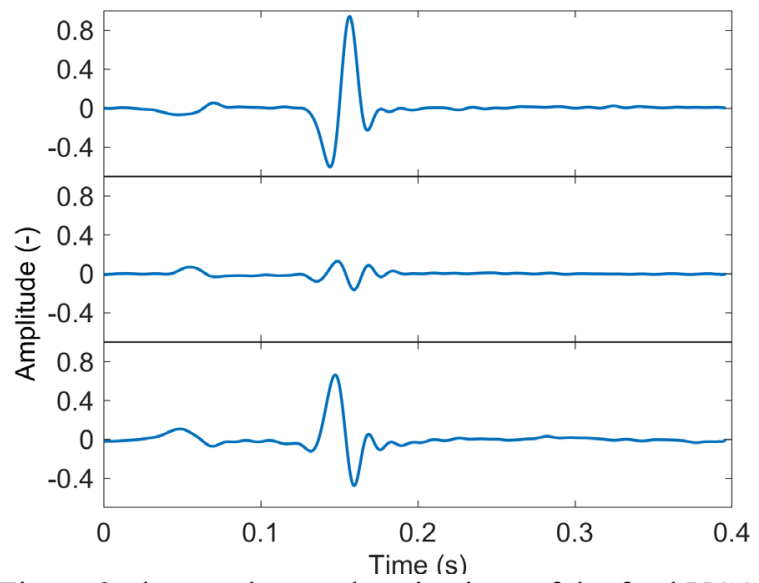

Figure 2: three orthogonal projections of the fetal VCG for one of the patients from the healthy cohort. The fetal orientation with respect to the recording electrodes is unknown.

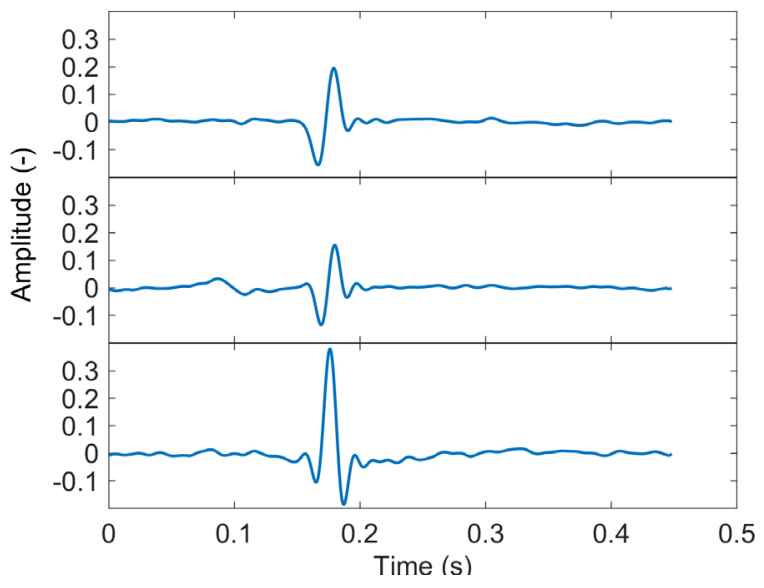

Figure 3: three orthogonal projects of the fetal VCG from a patient suffering from tetralogy of Fallot. The fetal orientation with respect to the recording electrodes is unknown.

The accuracy of the network in classifying fetal VCGs as healthy or CHD in the test set was $72 \%$.

\section{Discussion}

In this paper, a method was presented for computerized detection of CHD based on non-invasive fetal electrocardiography at around 20 weeks of gestation. The presented method requires 250 heartbeats to calculate a fetal VCG, which typically means that only about 2 minutes of data is necessary. Integration of the technology in the current prenatal screening should therefore be relatively easy. Especially since no expertise is required to perform the measurements and only in $2 \%$ of recordings the fully automated method failed to detect a fetal VCG.

It should be noted that the use of fetal electrocardiography for CHD detection, once further optimized, is not intended to replace the current ultrasonic examination. In fact, electrocardiography reveals information on the electrical system of the heart, while ultrasound reveals information about the mechanical activity. Although connected, the combination of electrical and mechanical activity provides a more comprehensive picture of the heart, hopefully leading to improved CHD detection rates.

With an accuracy of $76 \%$ in detecting CHD, the presented method outperforms global results for ultrasonic examination that are found in literature. Although this emphasizes the potential of the technology, it should be stressed that the dataset used in this study is not fully representative for a screening population. Patients in the CHD cohort were enrolled based on a positive CHD finding during prenatal screening. Hence it can be argued that in this dataset ultrasonic examination had perfect accuracy.

Another consequence of the dataset used in this study is that severe CHDs might be overrepresented. Subtle CHDs are more likely to be missed during the ultrasoundbased screening. However, severe CHD that show distinct abnormalities on the ultrasound might not always be accompanied by severe electrical abnormalities. A transposition of the great arteries, for instance, is expected to lead to only marginal electrical defects. Vice versa, subtle mechanical abnormalities might be associated with large electrical abnormalities. This stresses once more that both echocardiography and electrocardiography should be complimentary when it comes to CHD detection. This also stresses the need for a more thorough study to evaluate the potential of electrocardiography in a more general screening population and its potential in differential diagnostics.

\section{Conclusion}

The proposed method for non-invasive fetal electrocardiography followed by a deep neural network is able to detect congenital heart disease at 20 weeks of gestation with an accuracy of $76 \%$. This accuracy is higher than those reported in literature for echocardiography during prenatal screening. It should be noted however that, albeit promising, our result was obtained in a dataset that is not fully representative for the general screening population and further research towards the potential of fetal electrocardiography for CHD detection is required. 


\section{References}

[1] H. Dolk, M. Loane, E. Garne, and European Surveillance of Congenital Anomalies (EUROCAT) Working Group, "Congenital heart defects in Europe: prevalence and perinatal mortality, 2000 to 2005.," Circulation, vol. 123, no. 8, pp. 841-9, Mar. 2011.

[2] V. Fesslova' et al., "Changed outcomes of fetuses with congenital heart disease," J. Cardiovasc. Med., vol. 16, no. 8, pp. 568-575, Aug. 2015.

[3] H. Razzaghi, M. Oster, and J. Reefhuis, "Long-term outcomes in children with congenital heart disease: National Health Interview Survey," J. Pediatr., vol. 166, no. 1, pp. 119-124.e1, Jan. 2015.

[4] D. Bonnet et al., "Detection of transposition of the great arteries in fetuses reduces neonatal morbidity and mortality," Circulation, vol. 99, no. 7, pp. 916-918, Feb. 1999.

[5] W. T. Mahle, R. R. Clancy, S. P. McGaurn, J. E. Goin, and B. J. Clark, "Impact of prenatal diagnosis on survival and early neurologic morbidity in neonates with the hypoplastic left heart syndrome.," Pediatrics, vol. 107, no. 6, pp. 1277-82, Jun. 2001.

[6] W. Tworetzky, D. B. McElhinney, V. M. Reddy, M. M. Brook, F. L. Hanley, and N. H. Silverman, "Improved surgical outcome after fetal diagnosis of hypoplastic left heart syndrome.," Circulation, vol. 103, no. 9, pp. 126973, Mar. 2001.

[7] O. Franklin, "Prenatal diagnosis of coarctation of the aorta improves survival and reduces morbidity," Heart, vol. 87, no. 1, pp. 67-69, Jan. 2002.

[8] K. Mäkikallio et al., "Fetal aortic valve stenosis and the evolution of hypoplastic left heart syndrome: Patient selection for fetal intervention," Circulation, vol. 113, no. 11, pp. 1401-1405, 2006.

[9] C. L. van Velzen et al., "Prenatal detection of transposition of the great arteries reduces mortality and morbidity.," Ultrasound Obstet. Gynecol., vol. 45, no. 3, pp. 320-5, Mar. 2015.

[10] C. L. Van Velzen et al., "Prenatal detection of congenital heart disease - Results of a national screening programme," BJOG An Int. J. Obstet. Gynaecol., vol. 123, no. 3, pp. 400-407, Feb. 2016.

[11] D. J. Levy et al., "Improved prenatal detection of congenital heart disease in an integrated health care system," Pediatr. Cardiol., vol. 34, no. 3, pp. 670-679, Mar. 2013.

[12] J. Marek, V. Tomek, J. Škovránek, V. Povýšilová, and M. Šamánek, "Prenatal ultrasound screening of congenital heart disease in an unselected national population: A 21-year experience," Heart, vol. 97, no. 2, pp. 124-130, Jan. 2011.

[13] G. K. SHARLAND and L. D. ALLAN, "Screening for congenital heart disease prenatally. Results of a $2^{1 / 2} 2$-year study in the South East Thames Region," BJOG An Int. J. Obstet. Gynaecol., vol. 99, no. 3, pp. 220-225, 1992.

[14] I. Stümpflen, A. Stümpflen, M. Wimmer, and G. Bernaschek, "Effect of detailed fetal echocardiography as part of routine prenatal ultrasonographic screening on detection of congenital heart disease," Lancet, vol. 348, no. 9031, pp. 854-857, Sep. 1996.
[15] J. Behar, F. Andreotti, S. Zaunseder, J. Oster, and G. D. Clifford, "A practical guide to non-invasive foetal electrocardiogram extraction and analysis.," Physiol. Meas., vol. 37, no. 5, pp. R1-R35, 2016.

[16] R. Vullings, "Non-invasive fetal electrocardiogram: analysis and interpretation," 2010.

[17] L. A. Hamilton, E. Fisher, C. Horn, I. DuBrow, and D. Vidyasagar, "A new prenatal cardiac diagnostic device for congenital heart disease.," Obstet. Gynecol., vol. 50, no. 4, pp. 491-4, Oct. 1977.

[18] B. Brambati and L. Bonsignore, "The significance of indirect electrocardiography in fetal cardiac arrhythmias.," Eur. J. Obstet. Gynecol. Reprod. Biol., vol. 14, no. 6, pp. 371-3, Mar. 1983.

[19] C. Velayo et al., "Understanding congenital heart defects through abdominal fetal electrocardiography: Case reports and clinical implications," J. Obstet. Gynaecol. Res., vol. 37, no. 5, pp. 428-435, May 2011.

[20] S. Siddiqui, A. Wilpers, M. Myers, J. D. Nugent, W. P. Fifer, and I. A. Williams, "Autonomic regulation in fetuses with congenital heart disease," Early Hum. Dev., vol. 91, no. 3, pp. 195-198, Mar. 2015.

[21] B. Yilmaz, H. K. Narayan, A. Wilpers, C. Wiess, W. P. Fifer, and I. A. Williams, "Electrocardiographic intervals in foetuses with CHD," Cardiol. Young, vol. 26, no. 1, pp. 84-89, Dec. 2014.

[22] R. Vullings, C. H. L. Peters, R. J. Sluijter, M. Mischi, S. G. Oei, and J. W. M. Bergmans, "Dynamic segmentation and linear prediction for maternal ECG removal in antenatal abdominal recordings," Physiol. Meas., vol. 30, no. 3, pp. 291-307, 2009.

[23] G. J. J. Warmerdam, R. Vullings, L. Schmitt, J. O. E. H. Van Laar, and J. W. M. Bergmans, "A fixed-lag Kalman smoother to filter power line interference in electrocardiogram recordings," IEEE Trans. Biomed. Eng., vol. 64, no. 8, pp. 1852-1861, Aug. 2017.

[24] G. J. J. Warmerdam, R. Vullings, L. Schmitt, J. O. E. H. Van Laar, and J. W. M. Bergmans, "Hierarchical probabilistic framework for fetal R-peak detection, using ECG waveform and heart rate information," IEEE Trans. Signal Process., vol. 66, no. 16, pp. 4388-4397, 2018.

[25] R. Vullings, C. H. L. Peters, I. Mossavat, S. G. Oei, and J. W. M. Bergmans, "Bayesian approach to patienttailored vectorcardiography," IEEE Trans. Biomed. Eng., vol. 57, no. 3, pp. 586-595, Mar. 2010.

[26] R. Vullings, M. Mischi, S. Guid Oei, and J. W. M. Bergmans, "Novel bayesian vectorcardiographic loop alignment for improved monitoring of ECG and fetal movement," IEEE Trans. Biomed. Eng., vol. 60, no. 6, pp. 1580-1588, 2013.

Address for correspondence:

Rik Vullings

P.O. Box 513, $5600 \mathrm{MB}$, Eindhoven, The Netherlands r.vullings@tue.nl 